\title{
Symptomatic Sinus Node Disease: Natural History After Permanent Ventricular Pacing*
}

\author{
ARTHUR B. SIMON and ALLAN E. ZLOTO \\ From the Department of Internal Medicine, Division of Cardiology, University of Michigan \\ Medical Center, Ann Arbor, Michigan, U.S.A.
}

\begin{abstract}
SIMON, A.B., AND ZLOTO, A.E. Symptomatic sinus node disease: Natural history after permanent ventricular pacing. Fifty-nine patients between the ages of 13 and 88 with sinus node disease, who received a permanent ventricular pacemaker between 1965 and 1976 at one institution, were followed to determine the natural history of the disorder after permanent pacing. Nineteen had ischemic heart disease, six had primary myocardial disease, and eight valvular heart disease. In 26 , no etiology for the arrhythmia was apparent. The one- and five-year survival was $85.5 \%$ and $73.1 \%$, respectively. Patients with underlying heart disease had a significantly poorer survival when compared to those without $(58 \%$ versus $94 \%$ at 36 months), and all but 3 of 13 deaths in the first 36 months were in those with ischemic heart disease. There was a distinct trend toward poor survival in those with heart failure prior to pacemaker implant and those over age 65. Patients with sinus bradycardia alone did best ( $91 \%$ survival three years after implant), while those with bradycardia-tachycardia syndrome and those with sinoatrial arrest alone did distinctly worse $(76 \%$ and $65 \%$ survival at three years, respectively). Twelve of 18 deaths were due to progression of underlying heart disease. The long-term prognosis with symptomatic sinus node disease can be predicted in part by (1) etiology of the underlying heart disease, (2) pre-implant arrhythmia, and (3) ventricular function prior to implant. (PACE, Vol. 2, May-June, 1979)
\end{abstract}

bradycardia-tachycardia syndrome, patient longevity

Sinus node disease is manifested by either intermittent or persistent severe sinus bradycardia, sinoatrial block or periods of sinoatrial arrest. ${ }^{1-3}$ In its symptomatic stage, patients may have pre-syncope or syncope palpitations due to supraventricular tachyarrhythmias or congestive heart failure. ${ }^{4,5}$ Sinus node disease may be associated with congenital, valvular, myo-

Address for reprints: Arthur B. Simon, M.D., Heart Station, University Hospital, 1405 E. Ann Street, Ann Arbor, Michigan 48109, U.S.A.

*Supported in part by a grant from the Michigan Heart Association. pathic, or ischemic heart disease, but many patients have no apparent etiology. ${ }^{6}, 7$ The course of untreated symptomatic sinus node disease is unknown, because permanent pacing was used for symptom palliation prior to the availability of natural history studies. Frequently, pacing must be combined with treatment of congestive failure or tachyarrhythmias for maximium palliation of symptoms., ${ }^{45}$ This report describes the natural history of symptomatic patients with sinus node disease who have received a ventricular pacemaker at one institution, and elucidates factors which correlate with late mortality and morbidity. 


\section{Methods}

\section{Patient Population}

All adult patients who received their initial permanent pacemaker implantation for symptomatic sinus node disease at the University of Michigan Medical Center between July 1, 1965 and June 30, 1978 constitute the subjects of this report. Six patients with both sinus node dysfunction and atrioventricular block were excluded from this analysis and are described elsewhere. ${ }^{\circ}$ Seven patients who had previously received their initial pacemaker elsewhere for this indication but were subsequently followed ${ }^{\circ}$ here were also excluded. All patients were paced from the ventricle. The diagnosis of sinus node disease was made on clinical and electrocardiographic grounds, ${ }^{1,3,7}$ and corroborated by electrophysiologic testing in some. The diagnosis for inclusion in this study in the patients paced prior to the description of the syndrome ${ }^{1}$ was made retrospectively during a systematic evaluation of all patients receiving a permanent pacemaker in this institution. ${ }^{8}$ Selected variables from the history, clinical course, sequential electrocardiograms, including Holter monitoring before and after pacemaker insertion, and follow-up information were transferred to computer tape and analyzed using methods previously described. ${ }^{\text {All }}$ patients were assessed systematically at intervals no longer than six months. Special attention was paid to the atrial and underlying ventricular escape mechanism with pacemaker inhibition or at the time of pulse generator replacement. Survival curves were calculated according to the life table method using the Michigan Interactive Data Analysis System (MIDAS) ${ }^{9}$ and the Interactive Graphic Survival Analysis System (IGSAS). ${ }^{10}$ Survival time for each patient extended from the date of initial pacemaker implant until death or June 30, 1978. The Breslow's generalized Kruskal-Wallis Test was utilized to determine if the survival distribution differed across strata for any single pre-implant variable. ${ }^{11}$

The etiology of the underlying heart disease and arrhythmias complex in each patient was classified according to clinical data at the time of initial pacemaker implant. Arteriosclerotic heart disease was accepted as etiologic only if the patient had a past history of definite angina pectoris, or ECG or enzyme evidence of prior myocardial infarction. Valvular heart disease or primary cardiomyopathy was diagnosed according to recognized clinical and catheterization critieria. Patients without apparent underlying cardiac disease were classified as idiopathic'. The method for the determination of the cause of death was similar to that previously described. ${ }^{8,9}$ When more than one etiology of heart disease was identified classification was according to the clinically dominant type at entry. Details concerning the circumstances of death were obtained by interview of physician, family member, or other informed witnesses. Sudden death was defined as death within one hour after the onset of symptoms. Congestive heart failure was diagnosed utilizing the criteria of McKee. ${ }^{12}$ Chronic atrial fibrillation was defined as the presence of this arrhythmia on three successive electrocardiograms at least one year apart. During the time of the study, patients were paced from the ventricle at rates between 68 and 72 beats/minute with either General Electric, ${ }^{*}$ Medtronic, ${ }^{* *} \mathrm{CPI}^{* * *}$ or Cordisł pacing systems.

\section{Results}

Between 1965 and 1978, 59 adult patients received their initial permanent pacemaker for treatment of sinus node disease at the University of Michigan. Only 10 had their initial implant prior to 1970 . Although representing only $16 \%$ of all patients who have received permanent pacemakers at this institution since 1961, this has been the indication for pacing for $30 \%$ of all patients who received their initial unit since 1972. The mean follow-up period was $\mathbf{5 0}$ months (range: 1-120 months). One patient has been lost to follow-up. There were 33 men and 26 women. They ranged in age from 13 to 88 . The

\footnotetext{
${ }^{*}$ General Electric,

${ }^{\star *}$ Medtronic, Inc., Minneapolis, Minn., U.S.A.

${ }^{\star \star \star}$ Cardiac Pacemakers, Inc., St. Paul, Minn., U.S.A.

$\ddagger$ Cordis Corp., Miami, Florida, U.S.A.
} 
Table I.

Correlation Between Clinically Apparent Etiology of the Patients' Heart Disease and Selected Clinical Features Before Pacemaker Implant. The Idiopathic Group had Significantly Less Coexistent IIIness

\begin{tabular}{|c|c|c|c|c|c|}
\hline & $\begin{array}{l}\text { Idiopathic } \\
n=26\end{array}$ & $\begin{array}{l}\text { Ischemic } \\
n=19\end{array}$ & $\begin{array}{l}\text { Valvular } \\
n=8\end{array}$ & $\begin{array}{c}\text { Cardio- } \\
\text { myopathy } \\
n=6\end{array}$ & $\begin{array}{l}\text { Total } \\
n=59\end{array}$ \\
\hline Angina pectoris & - & 9 & - & - & $9(15 \%)$ \\
\hline Myocardial infarction & - & 12 & $\dot{-}$ & - & $12(20 \%)$ \\
\hline Congestive heart failure & 2 & 11 & 2 & 1 & $16(27 \%)$ \\
\hline Diabetes mellitus & 5 & 13 & 1 & 5 & $24(41 \%)$ \\
\hline Systolic hypertension & 5 & 8 & - & 1 & $14(24 \%)$ \\
\hline Diastolic hypertension & 5 & 6 & - & - & $11(19 \%)$ \\
\hline Obesity & 8 & 10 & - & 4 & $22(37 \%)$ \\
\hline COPD & 2 & 4 & - & 2 & $8(14 \%)$ \\
\hline Renal insufficiency & 5 & 5 & 1 & 2 & $13(22 \%)$ \\
\hline Malignant neoplasm & 3 & 2 & - & - & $5(8 \%)$ \\
\hline $\begin{array}{l}\text { Peripheral arterial } \\
\text { insufficiency }\end{array}$ & 3 & 3 & - & 2 & $8(14 \%)$ \\
\hline Hypercholesterolemia & 4 & 2 & - & - & $6(10 \%)$ \\
\hline Stroke & 2 & 3 & - & - & $5(8 \%)$ \\
\hline
\end{tabular}

COPD $=$ Chronic obstructive pulmonary disease

mean age at initial implant was 64.6 years. Thirty-eight were over age 65 . The clinical features of the entire group are summarized in Table I. There were 19 patients with ischemic heart disease, of whom three also had hypertensive heart disease and four mitral insufficiency attributed to papillary muscle dysfunction. Six patients had primary myocardial disease, and eight had valvular disease. One of these has mitral valve prolapse with long QT syndrome, one isolated aortic stenosis, five rheumatic valve disease, and one patient has a tricuspid valve replacement secondary to endocarditis. Twenty-six patients had no apparent cardiac disease except the arrhythmia. Two patients in this latter group had quadriplegia or hemiplegia from spinal cord trauma.

\section{Previous Status}

\section{Coexistent Disease Before Implant}

There was a high prevalence of chronic diseases in this population. Twenty-four had diabetes mellitus, 14 had systolic ( $>160 \mathrm{~mm} \mathrm{Hg}$ ) and 11 diastolic ( $>95 \mathrm{~mm} \mathrm{Hg}$ ) hypertension (as determined on at least two blood pressure determinations); obesity was present in 22 , chronic obstructive pulmonary disease in eight, and renal insufficiency in 13; six of the 36 men had obstructive uropathy; five patients had a past history of malignant neoplasm. The prevalence rate of diabetes mellitus far exceeds that in the general population,,$^{13}$ and is higher than in patients with atrioventricular block previously reported from this institution. ${ }^{8}$ Five patients had a definite stroke prior to pacemaker implant. 'Two patients had carotid bruits. Organic brain syndrome, Parkinson's disease and previous saddle embolus were diagnosed in single patients.

\section{Cardiac Status Before Implant}

Sinus bradycardia alone, either in combination with congestive failure incompletely responsive to medical therapy (five patients) or with syncope historically without documenta- 
Table II.

Correlation Between the Three Major Subgroups of the Population Evaluated and Underlying Etiology of Heart Disease

\begin{tabular}{lccccc}
\hline & Idlopathic & lschemic & Valvular & $\begin{array}{c}\text { Cardio- } \\
\text { myopathy }\end{array}$ & Total \\
\hline $\begin{array}{l}\text { Sinus bradycardia } \\
\text { alone }\end{array}$ & $4(15 \%)$ & $2(11 \%)$ & $2(25 \%)$ & $2(33 \%)$ & 16 \\
$\begin{array}{l}\text { Sinoatrial arrest and } \\
\text { sinus bradycardia }\end{array}$ & $15(58 \%)$ & $11(58 \%)$ & $2(25 \%)$ & $2(33 \%)$ & 30 \\
$\begin{array}{c}\text { Bradycardia-tachycardia } \\
\text { syndrome }\end{array}$ & $7(27 \%)$ & $6(32 \%)$ & $4(50 \%)$ & $2(33 \%)$ & 19 \\
\begin{tabular}{l} 
Total \\
\hline
\end{tabular} & $26(44.1 \%)$ & $19(32.2 \%)$ & $8(13.6 \%)$ & $6(10.2 \%)$ & 59 \\
\hline
\end{tabular}

tion of sinoatrial block (five patients), was seen in ten. Characteristically, rates under $50 / \mathrm{min}$ were seen and, in most cases, rates failed to exceed $70 /$ min with moderate exercise. Sinoatrial block or arrest ${ }^{14}$ in combination with sinus bradycardia was seen in 30 patients; 19 patients were classified as having "bradycardia-tachycardia syndrome". Twelve of these had atrial fibrillation or flutter, two had multifocal atrial tachycardia and five had atrial tachycardia. There was no correlation between the etiology of heart disease and the type of arrhythmia seen (Table II).

Ventricular premature beats were also common. Twelve patients had unifocal, and two had multifocal PVCs during their pre-implant evaluation; seven patients had one or more episodes of ventricular tachycardia, and one patient had been resuscitated from ventricular fibrillation prior to pacemaker implant. Left bundle branch block was present in four, isolated right bundle branch block in two, left anterior fascicular block in two, right bundle branch block with left anterior fascicular block in three, alternating right and left bundle branch block in one, and prolonged PR interval in 15.

Sixteen patients had congestive heart failure in combination with their arrhythmia. Eleven patients had mild, and eleven had marked cardiomegaly by chest roentgenogram. There was a wide variability in the duration of symptoms among all patients, regardless of etiology of heart disease. Twenty-four patients had symptoms of heart disease or arrhythmias for less than one year, but 18 patients had symptoms for over five years. The median duration of symptoms for patients in the idiopathic group was 12 months (range: < 1 month - 18 years), while those with ischemic heart disease had a median duration of symptoms of 54 months. Forty-six of the 59 patients had presyncope or syncope; 23 described these symptoms for over 12 months; nine of these had described such episodes for eight years or more.

\section{Course Following Pacemaker Implant Mortality}

Overall survival and the correlation between survival and underlying cardiac disease is shown in Figure 1. Eighteen patients have died in the follow-up period. The six-month, oneyear and five-year survival was $91 \%, 85.5 \%$, and $73.1 \%$, respectively. Early deaths were usually cardiac, while late deaths were usually due to non-cardiac causes. Mortality was significantly higher in those with ischemic heart disease $(\mathrm{p}<$ 0.02) (Fig. 1), and tended to be higher in those with congestive heart failure prior to implant ( $p$ $=0.07$ ). Mortality was very low in those with sinus bradycardia alone $85.9 \%$ survival at three years), but significantly greater in those with sinoatrial arrest or bradycardia-tachycardia syndrome $(64.8 \%$ and $75.6 \%$, respectively) ( $\mathrm{p}<$ .05) (Fig. 2). There was a poorer correlation be- 


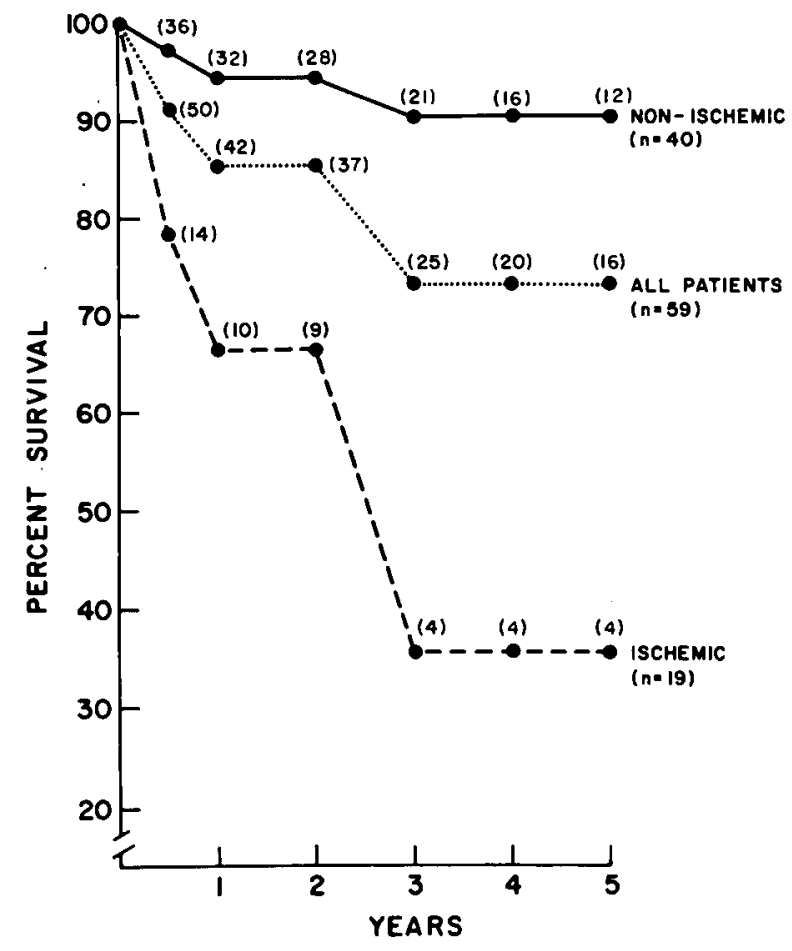

Figure 1. Correlation between survival and etiology of underlying cardiac disease to 60 months of follow-up using the life table method. Five patients, two with cardiomyopathy, one with rheumatic heart disease, and two with no cardiac disease, died 61 to 96 months post-implant and are not presented on the survival curves. Numbers in parenthesis indicate numbers of patients at risk at each interval.

tween survival and age. The five-year survival for those less than 65 years old was $74 \%$, and $63 \%$ for those over 65 (N.S., $p=0.17$ ). The mortality exceeded that of sex- and age-matched controls from the general U.S. population. ${ }^{8}$

Progressive and refractory congestive heart failure was a common cause of death; three of 18 deaths in this series were primarily attributed to heart failure (Table III). Six additional patients died of definite (unequivocal ECG, enzyme or autopsy data) or probable (characteristic history but no autopsy confirmation) acute myocardial infarction. Two patients with ischemic heart disease died of an acute myocardial infarction and shock within five days after the initial transvenous pacemaker

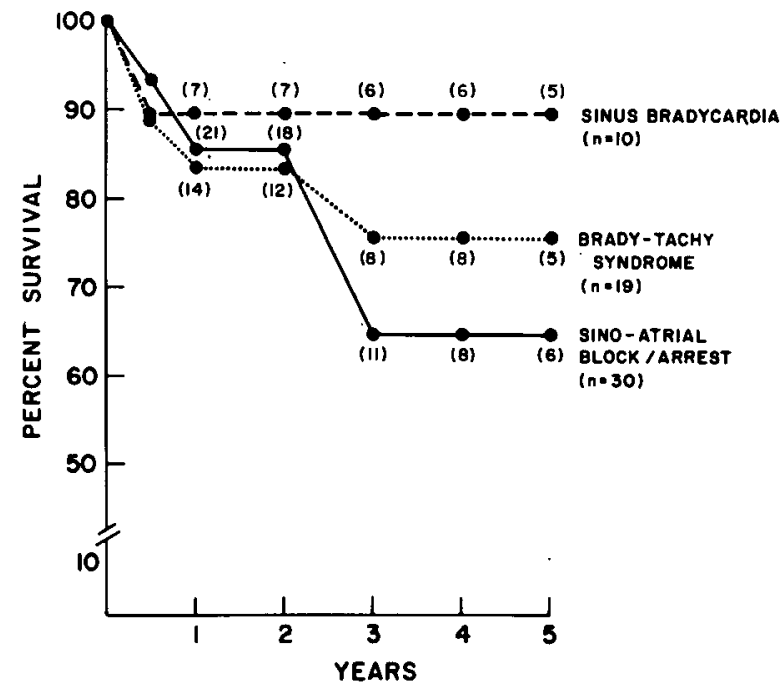

Figure 2. Correlation between survival and arrhythmia prior to implant. Four patients with sinoatrial arrest and one patient with bradycardia-tachycardia syndrome died 61 to 96 months post-implant and are not presented on the survival curves. Numbers in parenthesis indicate numbers of patients at risk at each interval.

implantation. Three patients died suddenly, two of whom had ventricular tachycardia before implant. Therefore, at least 12 of the 18 deaths were due to cardiac events. In three patients, insufficient data was available to determine the immediate cause of death, and two patients died of pulmonary causes. Nine deaths occurred in the hospital and three in convalescent facilities; eight patients were autopsied. In no patient did pacemaker malfunction contribute to death.

\section{Morbidity}

Although one of the recognized indications for permanent pacemaker implantation is to improve symptoms of congestive failure in patients with slow heart rates, it was necessary to continue digitalis or diuretics during follow-up in all but one patient with heart failure before implant. However, symptoms generally improved and overall management was simplified in most after pacing; five additional pa- 
Table III.

Correlation Between Primary Cause of Death and Underlying Etiology of Heart Disease as Determined at the Time of Initial Implant

\begin{tabular}{|c|c|c|c|c|c|}
\hline - & $\begin{array}{l}\text { Idiopathic } \\
\quad n=26\end{array}$ & $\begin{array}{l}\text { Ischemic } \\
n=19\end{array}$ & $\begin{array}{c}\text { Valvular } \\
n=8\end{array}$ & $\begin{array}{c}\text { Cardio- } \\
\text { myopathy } \\
n=6\end{array}$ & $\begin{array}{l}\text { Total } \\
\mathrm{n}=\mathbf{5 9}\end{array}$ \\
\hline Congestive heart failure & - & 2 & - & 1 & 3 \\
\hline Myocardial infarction & 1 & 5 & - & - & 6 \\
\hline Sudden death & - & - & 2 & 1 & 3 \\
\hline Noncardiac death & 1 & 1 & 1 & - & 3 \\
\hline $\begin{array}{l}\text { Uriknown cause } \\
\text { (unwitnessed) }\end{array}$ & 1 & 1 & - & 1 & 3 \\
\hline Total mortality. & $3(11.6 \% *)$ & $9(47.4 \%)$ & $3(37.5 \% *)$ & $3(50.0 \% ")$ & $18(30.5 \% ")$ \\
\hline
\end{tabular}

"Percent is expressed as the percent mortality in each etiologic subgroup.

tients have required treatment for chronic congestive heart failure following pacemaker implantation; in at leảst three this appeared following a definite or probable myocardial infarction. One patient with mitral valve prolapse, sinus bradycardia, long QT syndrome, and ventricular tachycardia has been resuscitated from ventricular fibrillation.

In addition to the major central nervous system events before implant, during follow-up two patients have had a complete stroke and three have had transient ischemic attacks; progressive chronic brain syndrome occurred in two. Progressive and severe renal insufficiency was seen in three, and four patients have had major pulmonary complications (either pulmonary insufficiency or pulmonary embolus) which resulted in death in two of these. One patient has had a new malignancy.

\section{Arrhythmias in the Late Follow-up Period}

In addition to the resuscitated patient, 16 of the patients have had elective 24-hour ambulatory monitoring on a random basis 1 to 108 months following initial pacemaker implant. Only two had no PVCs; eight had < 1 PVC/1000 normal or paced beats, but four $(25 \%)$ had 1 to $10 \mathrm{PCVs}$ and three $(18 \%)$ have $>30 \mathrm{PVCs} / 1000$ normal beats. Ten of the 14 patients with PVCs have multiformed PVCs, and three have couplets; two have had sustained ventricular tachycardia noted during routine monitoring, which has resulted in the addition of antiarrhythmic agents to their present drug program. Both of these latter patients are in the cardiomyopathy group. There was no correlation between the severity of the ventricular arrhythmias noted and the etiology of the underlying heart disease.

In two patients in this series, pacing was discontinued one and one-half and four years after implant without return of symptoms. One of the patients with bradycardia-tachycardia syndrome refused pacemaker replacement. Four years later, he was asymptomatic despite the fact that his pacemaker had ceased functioning. Mild sinus bradycardia persists. Pacing was cautiously discontinued 18 months following implant in a young woman with spinal cord trauma and sinoatrial block whose initial pacemaker required removal because of pulse generator erosion. Her bradyarrhythmia has not returned.

The long term follow-up of the pattern of supraventricular arrhythmias is summarized in Table IV. Seven of the 19 patients (37\%) with bradycardia-tachycardia syndrome have converted to chronic atrial fibrillation during follow-up. Conversion occurred from 11 to 60 months after implant (mean: 38.6 months). No patient with isolated sinus bradycardia or sinoatrial arrest has developed chronic or recur- 
Table IV.

Correlation Between Mechanism of Supraventricular Arrhythmia Seen Pre-implant and Supraventricular Mechanism During the Follow-up Period Within the Bradycardia-Tachycardia Subgroup

\begin{tabular}{|c|c|c|c|c|}
\hline \multirow[b]{2}{*}{$\begin{array}{l}\text { Pre-implant } \\
\text { Supraventricular } \\
\text { Arrhythmias }\end{array}$} & \multicolumn{4}{|c|}{ Post-Implant Supraventricular Mechanism } \\
\hline & $\begin{array}{c}\text { Short } \\
\text { Follow-up or } \\
\text { or Early Death }\end{array}$ & $\begin{array}{c}\text { Continued } \\
\text { Intermittent } \\
\text { Atrial } \\
\text { Arrhythmias }\end{array}$ & $\begin{array}{c}\text { Chronic } \\
\text { Atrial } \\
\text { Fibrillation }\end{array}$ & Total \\
\hline $\begin{array}{l}\text { Atrial fibrillation } \\
\text { or atrial flutter }\end{array}$ & 3 & 4 & 5 & 12 \\
\hline $\begin{array}{c}\text { Multifocal atrial } \\
\text { tachycardia }\end{array}$ & - & - & 2 & 2 \\
\hline Atrial tachycardia & 1 & 3 & 1 & 5 \\
\hline Total & 4 & 7 & 8 & 19 \\
\hline
\end{tabular}

rent supraventricular arrhythmias during follow-up although the one patient with sinus bradycardia, mitral valve prolapse, and long QT syndrome has exercise-induced atrial fibrillation and atrial tachycardia, and two patients with sinoatrial arrest have had isolated episodes of atrial fibrillation. In two patients $(3.8 \%)$, high grade AV block was documented at the time of pacemaker inhibition in the clinic within one year of initial implant.

\section{Discussion}

There is a wide variation in the reported mortality in patients with sinus node disease. In the series by Rokseth and Hatle, ${ }^{15}$ Rubenstein et al, ${ }^{3}$ Conde et $a l,{ }^{5}$ and Aroesty et $a,^{16}$ death was reported to be infrequent following pacemaker implant. Krishaswami and Geraci," and Wohl et al, ${ }^{18}$ however, have observed a significant mortality, especially within the first two years after pacemaker insertion, and similar results are seen in this study. In part, the reported differences in survival can be attributed to differences in patients selection. Conde et al, ${ }^{5}$ for example, excluded all patients who had had an acute myocardial infarction within one year. Such patients had a poor prognosis in the present series. Others have included patients only in the bradycardia-tachycardia subgroup of this syndrome..$^{4,5,18,19}$ Wohl et al ${ }^{18}$ excluded those lost to follow-up, and Wan et $\mathrm{al}^{20}$ and Rubenstein et $\mathrm{al}^{3}$ included many asymptomatic patients not permanently paced. The age group from which the patients are drawn may also play some role. The age of the group is slightly older than that reported by Radford and Julian, 7 but younger than others. ${ }^{5}$

It is quite clear from this series that significant mortality exists in this population, but that mortality, particularly early mortality, is highest in the ischemic heart disease subgroup. For example, six of the 19 patients with ischemic heart disease died within the first 12 months after pacemaker implant, while only two deaths occurred in the remaining 40 patients without ischemic heart disease. It should be noted, however, that those in the ischemic heart disease subgroup were also slightly older than the patients with idiopathic, valvular, or primary myocardial disease, and had a higher prevalence of diabetes mellitus, hypertension, chronic lung disease, and congestive heart failure. While none of these factors alone could be demonstrated to influence mortality in a statistically significant way in this small study group, their combined influence may have contributed to overall mortality. The pattern of mortality was similar to that reported by Fruehan et al. ${ }^{21}$

Persistent ventricular arrhythmias during follow-up were common, but their presence did not appear to correlate with underlying cardiac 
disease. Of particular interest was the high rate of conversion of patients with symptomatic supraventricular arrhythmias prior to implant to chronic atrial fibrillation during the followup period. In fact, no patient with only sinus bradycardia or periods of sinoatrial arrest without supraventricular arrhythmias has progressed to chronic atrial fibrillation during follow-up, although two have had transient atrial fibrillation. Amikam and Riss ${ }^{22}$ reported that seven of 25 patients with sick sinus syndrome developed chronic atrial fibrillation, but this was noted in both the bradyarrhythmia and the bradycardia-tachycardia subgroups. Vera et $\mathrm{al}^{23}$ have previously reported that 11 of 40 patients in their/series had done likewise.It is apparent that the development of chronic atrial fibrillation is a common late development in patients with sinus node disease. It is uncertain whether patients in this subgroup will remain in atrial fibrillation indefinitely or require pacing at all at this time; in no such patient in this series has pacing been discontinued.

Pacing has been discontinued indefinitely, however, in two patients when the opportunity arose to re-evaluate them without a permanent pacemaker-once because of lack of patient cooperation in follow-up, and once because late infection dictated removal of the system and a period of observation. Such opportunities are uncommon, and periodic reassessment of the remaining patients in this series at the time of pacemaker inhibition in the clinic setting or at the time of pulse generator replacement would indicate that other than those who have converted to atrial fibrillation, there are few who would be likely to have had resolution of the bradyarrhythmia.

The patient with marked sinus bradycardia alone represents a difficult problem. When overt syncope historically accompanies the bradyarrhythmia, repeated ambulatory monitoring or electrophysiologic provocative studies may elucidate a possible mechanism for the syncope. Despite these efforts, five of the ten patients in this series with sinus bradycardia alone and syncope had no sustained asystolic event documented. Four have had resolution of syncopal episodes. One has persistent ventricu- lar arrhythmias requiring supression. The other five had overt congestive heart failure or severe activity restriction and sinus bradycardia. One died suddenly shortly following implant. One is essentially asymptomatic, although the need for occasional diuretics and pronounced cardiomegaly persists. The remaining three require continuous treatment for heart failure which is markedly improved since implant. It is obvious that this is not a homogeneous group of patients, but in all, management was simplified and symptoms improved following pacemaker implant.

Although intraventricular conduction disturbances are common prior to implant, ${ }^{24}$ in only two patients has atrioventricular block been noted during follow-up. One of these had a prolonged PR interval prior to implant. Previous studies have indicated that progression to atrioventricular block was uncommon in patients with sinus node disease paced from the coronary sinus. Moss et al ${ }^{25}$ reported that only one of 27 patients paced for sinus node disease developed atrioventricular block during follow-up, and this series is in agreement with that observation. Atrial or coronary sinus pacing, therefore, would appear to be a logical, and possibly beneficial, method of long-term pacing for the group of patients with congestive heart failure in combination with sinus bradycardia or sinoatrial arrest. There appears to be only a small probability that conversion to atrioventricular sequential or ventricular pacing would be required later in their course.

The survival after pacemaker insertion for sinus node disease is similar to the survival of patients paced for atrioventricular block previously reported from this institution during the same time period..$^{8}$ From this retrospective analysis of patients with sinus node disease, the presence of ischemic heart disease identifies a patient with a poor prognosis. A more favorable, but hardly benign long-term outlook, exists for the symptomatic group with heart disease of another etiology.

Progression of ventricular dysfunction, worsening underlying ventricular arrhythmias leading to sudden death, and a significant risk of cerebral emboli also appear common in this pa- 
tient population and contribute to morbidity and mortality. ${ }^{28}$ The result of this analysis suggests that long-term survival following pacemaker implantation in patients with symptomatic sinus node disease is correlated with a number of variables, including (1) the etiology of the underlying heart disease, (2) associated congestive heart failure, (3) age, and (4) the specific arrhythmias demonstrated prior to implant.

\section{References}

1. Ferrer, M.I.: The sick sinus syndrome in atrial disease. J.A.M.A., 206:645, 1968.

2. Easley, R.M., and Goldstein, S.: Sino-atrial syncope. Amer. J. Med., 50:166, 1971.

3. Rubenstein, J.J., Schulman, C.L., Yurchak, P.M., and DeSanctis, R.W.: Clinical spectrum of the sick sinus syndrome. Circulation, 46:5, 1972.

4. Moss, A.J., and Davis, R.J.: Brady-tachy syndrome. Prog. Cardiovasc. Dis., 16:438, 1974.

5. Conde, C.A., Leppo, J., Lipski, J., Stimmel, B., Litwak, R., Donoso, E., and Dack, S.: Effectiveness of pacemaker treatment in the bradycardia-tachycardia syndrome. Amer. J. Cardiol., 32:209, 1973.

6. Greenwood, R.D., Rosenthal, A., Sloss, L.J., LaCorte, M., and Nadas, A.: Sick sinus syndrome after surgery for congenital heart disease. Circulation, 52:208, 1975.

7. Radford, D.J., and Julian, D.G.: Sick sinus syndrome: Experience of a cardiac pacemaker clinic. Brit. Med. J., 3:504, 1974.

8. Simon, A.B., and Zloto, A.E.: Atrio-ventricular block: Natural history after permanent pacemaker implantation. Amer. J. Cardiol., 41:500, 1978.

9. Fox, D.J., and Guire, K.E.: Documentation for MIDAS (Michigan Interactive Data Analysis System), 2nd edition (revised). Statistical Research Laboratory. University of Michigan, Ann Arbor, Michigan, 1974.

10. Heilbrun, L.K.: Documentation of IGSAS (Interactive Graphic Analysis System), second edition. Department of Biostatistics, School of Public Health, University of Michigan, Ann Arbor, Michigan, 1975.

11. Breslow, N.: A generalized Kruskal-Wallis Test for comparing K samples subject to unequal patterns of censorship. Biometrika, 57:579, 1970.

\section{Acknowledgments}

The authors gratefully acknowledge the assistance of Drs. Leon Ostrander and Bertram Pitt for their critical review of the material, Mrs. Betty Plunkett for manuscript preparation, and Mr. Jamal A. Rassoul for his aid in data processing and analysis.

12. McKee, P.A., Castelli, W.P., and McNamara, P.M.: The natural history of congestive heart failure. The Framingham Study. New Eng. J. Med., 285:1441, 1971.

13. Davidson, D.M., Braak, C.A., Preston, T., and Judge, R.: Permanent ventricular pacing effect on long term survival, congestive heart failure and subsequent implantation. Ann. Intern. Med., 77:345, 1972.

14. Schamroth, L.: The Disorders of Cardiac Rhythm. Oxford, Blackwell Scientific Publications, 1971, p. 193.

15. Rokseth, R., and Hatle, L.: Prospective study on the occurrence and management of chronic sinoatrial disease, with followup. Brit. Heart J., 36:582, 1974.

16. Aroesty, J.M., Choen, S.I., and Morkin, E.: Bradycardia-tachycardia syndrome: Results in 28 patients treated by combined pharmacologic therapy and pacemaker implantation. Chest, 66:257, 1974.

17. Krishnaswami, V., and Geraci, A.R.: Permanent pacing in disorders of sinus node function. Amer. Heart J., 89:579, 1975.

18. Wohl, A.J., Laborde, J., Atkins, J.M., Blomquist, C.G., and Mullins, C.B.: Prognosis of patients permanently paced for sick sinus syndrome. Arch. Intern. Med., 136:406, 1976.

19. Kaplan, B.M., Langendorf, R., Lev, M., and Pick, A.: Tachycardia-bradycardia syndrome (socalled "sick sinus syndrome") pathology, mechanisms and treatment. Amer. J. Cardiol., 31:497, 1973 .

20. Wan, S.H., Lee, G.S., and Toh, C.C.S.: The sick sinus syndrome: A study of 15 cases. Brit. Heart J., 34:942, 1972.

21. Fruehan, W.F., Henegham, W.F., and Eich, R.H: Late mortality of patients with sick sinus syn- 


\section{SIMON AND ZLOTO}

drome. Circulation, 53-54 (Suppl):II-77, 1976 (abstr.).

22. Amikam, S., and Riss, E.: The natural history of sick sinus syndrome following permanent pacemaker implantation. Circulation, 55-56 (Suppl.):III-155, 1977 (abstr.).

23. Vera, Z., Mason, D., Awan, N., Janzen, D., Tonkon, M., and Vismara, L.: Spontaneous development of stable atrial fibrillation in patients with sick sinus syndrome. Circulation, 51-52 (Suppl.):II-93, 1975 (abstr.).
24. Rosen, K.M., Loeb, H.S., and Ziad, S.M.: Cardiac conduction in patients with symptomatic sinus node disease. Circulation, 43:836, 1971.

25. .Moss, A.J., Rivers, R.J., and Kramer, D.H.: Permanent pervenous atrial pacing from the coronary vein. Circulation, 49:22, 1974.

26. Fairfax, A.J., Lambert, C.D., and Lentham, A.: Systemic embolism in chronic sinoatrial disorders. New Eng. J. Med., 295:190, 1976. 
This document is a scanned copy of a printed document. No warranty is given about the accuracy of the copy. Users should refer to the original published version of the material. 